\title{
LA ENFERMERÍA, REALIDAD DE CIUDADANÍA Y DE GÉNERO EN CHILE
}

\section{NURSING, CITIZENSHIP AND GENDER REALITY IN CHILE}

\author{
Amaya PaVez Lizarraga*
}

\begin{abstract}
RESUMEN
Esta reflexión tiene por objetivo abordar la identidad de la enfermería en el contexto de la ciudadanía y el género. Se describe la importancia de los tres aspectos identidad, ciudadanía y género en la construcción de la realidad social, donde la enfermería como disciplina y profesión está sujeta al significado de estos conceptos, siendo simultáneamente una manifestación de la relación y el equilibrio que existe entre la realidad concreta y la realidad simbólica. Se concluye en la importancia de la consolidación de la enfermería en la dinámica de la sociedad actual, donde el cuidado emerge como un problema de sociedad que traspasa las fronteras del mundo privado y femenino.
\end{abstract}

Palabras clave: Enfermería, identidad de género, valores sociales, interacción social.

\begin{abstract}
This reflection aims to addresses the identity of nursing in the context of citizenship and gender. It describes the importance of the three aspects: identity, citizenship and gender, in the social construction of reality. Nursing as a discipline and profession is subject to the meaning of these concepts, being simultaneously a manifestation of the relationship and the balance existing between the factual and symbolic reality. We conclude on the importance of the consolidation of nursing in the dynamics of the contemporary society where caring emerge as a societal problem that oversteps the private and the feminine world.
\end{abstract}

Key words: Nursing, gender identity, social values, social interaction.

Fecha recepción: 20/12/11 Fecha aceptación: 12/08/13

* Enfermera-matrona, antropóloga social. Proyecto N 791220019 PAI Comisión Nacional de Investigación Científica y Tecnológica (CONICYT). Universidad de Santiago de Chile.Email: amaya.pavez@usach.cl 


\section{INTRODUCCIÓN}

El género es un concepto que permite la comprensión contemporánea del ser humano y su quehacer (1), circunscribiendo los espacios sociales, ordenando los comportamientos y modelando las posibilidades que la sociedad otorga a las personas -sujetos- y que se traducen en las expectativas personales y colectivas, en el estatus social y en las funciones asignadas para cada persona o grupo, proporcionando elementos para la construcción de significado de ciudadanía (2).

De tal forma que la ciudadanía y el género se imbrican y se potencian a través de diferentes mecanismos sociales y culturales, entre ellos las profesiones que son marcadas por el significado de estos conceptos en la construcción de la identidad profesional. Dicho de otro modo, estos aspectos son inherentes a las vivencias de las personas debido a que afectan directamente a la identidad individual y a la identidad social en los distintos espacios de la vida cotidiana, entre los cuales está el ejercicio profesional.

La identidad de la enfermería está construida alrededor de la feminidad (3) y de la responsabilidad social que tiene la mujer en occidente en el contexto de la modernidad, donde desde la tradición burguesa, la mujer, se inserta en el espacio privado y en la cotidianidad y cuya función principal es el trabajo reproductivo que se sostiene en el cuidado (4).

La visibilidad de la mujer en el espacio público se produce a través de la continuidad del cuidado que entrega a las personas necesitadas, desempeñando una función preferentemente de apoyo a las decisiones de los hombres en el contexto de la masculinidad hegemónica. En el caso de las mujeres enfermeras, éstas cuidan a las personas afectadas en su salud. De forma que la enfermería se constituye como una labor social donde la mujer contribuye a la sociedad cuidando a los enfermos y apoyando las acciones de los médicos.

Si bien lo anterior se inserta en el sentido hegemónico del sistema sexo-género, lo cierto es que la enfermería reconoce desde los inicios que su ontología no está en el apoyo al médico (5) si no en el cuidado de la persona necesitada. Esta diferencia de significados está marcada por una escisión en la identidad de enfermería entre dos representaciones, la social arraigada en la feminidad tradicional-hegemónica donde la enfermera se asimila a la mujer y la representación de la disciplina donde la enfermera es un agente de salud con una feminidad activa corporeizada en un sujeto social. Es aquí donde se instala la tensión entre hacer enfermería y pensar enfermería, la primera tiene un fuerte vínculo con el universo simbólico (6) de la masculinidad que se apoya en el quehacer naturalizado y de la cotidianidad. Un universo simbólico es aquel constructo que refiere un conjunto coherente de ideogramas cuyos significados están en correspondencia y conforman marcos de interpretación de la realidad. Los universos simbólicos se constituyen en mapas de significados y contenidos compartidos por los conglomerados sociales. La segunda, el pensar enfermería, se sitúa en el sentido de la feminidad y en el movimiento progresivo de empoderamiento y autonomía que se desarrolla desde el inicio de la modernidad hasta la actualidad.

En este proceso de identidad de las mujeres y de la enfermería podemos identificar la compenetración que existe entre estas tres dimensiones -identidad, ciudadanía y género- donde converge el aspecto social y el individual en la unidad del sujeto. Un sujeto situado en dos contextos, cada uno pudiendo tener dos niveles de análisis. El primer contexto es el espacio concreto representado por el hacer y por las instituciones, y el segundo es el espacio simbólico que ordena la realidad de forma imperceptible para la conciencia mediante relaciones de poder de expresión macro y microsocial.

Así, el sujeto es una persona mujer u hom- 
bre, organizado en una sociedad e inserto en una cultura que le proporciona un esquema de valores, creencias e ideología que orientan su comportamiento. Una persona con conciencia de sí misma que le permite la reflexión y la crítica, posibilitando la dinámica social y el cambio. Un sujeto que posee una identidad que se caracteriza por ser aquella dimensión en la cual se reconoce a sí mismo diferenciándose de los otros y que a su vez los otros lo reconocen en coherencia con la idea que tiene de sí mismo, produciéndose una armonía entre la autopercepción y la percepción social.

La ciudadanía es el espacio de acción donde las personas explicitan su pertenencia social y ejercen sus derechos y deberes políticos influyendo en las decisiones sociales (7) e interviniendo en la construcción de la sociedad. Y el género es la categoría de análisis (8) que permite comprender el sistema de relaciones sociales de poder que orientan los comportamientos y la identidad de los sujetos según los modelos culturales asignados a hombres y a mujeres.

Ahora bien, la ciudadanía, la identidad y el género estructuran al sujeto en una relación interdependiente y simultánea, semejante a esferas móviles que se acoplan y desacoplan conforme a la trayectoria de vida de cada persona. Estas tres dimensiones están compenetradas y una lleva a la otra. Hablar de ellas por separado es sólo en un afán de comprensión, debido a que así es posible analizar cómo repercute una sobre la otra, y al observar esto se ve, cómo se retroalimentan, se entrelazan o se anticipan (2).

Esta relación es parte de la vida social independiente de la especificidad de la experiencia, pudiendo observar la dinámica de la interrelación de estas tres dimensiones en la práctica de la enfermería, tanto en las personas que ejercen la profesión como en los receptores del cuidado, siendo una expresión más del mosaico de la realidad.

La estabilidad social necesita que la representación social coincida con la repre- sentación individual (9), así la enfermera/o ejerce la enfermería en consistencia con lo que las personas y la sociedad esperan que haga, porque la identidad que el medio le da a la enfermería debe ser similar a la autoidentidad de la enfermera/o y ésta debe ser compatible con el modelo de género imperante y con la autonomía de las decisiones que pueda tomar. Es decir, en la expresión de su ciudadanía, de lo contrario se produce un quiebre que amenaza la reproducción de la estabilidad social, provocando la reacción coercitiva del sistema ante la transgresión al modelo esperado, dando origen a un proceso dinámico de ajuste, reacción y desplazamiento de las fronteras culturales.

La crítica feminista señala que el género es transversal en la vida social, siendo el sistema sexo-género (10) el dispositivo cultural por excelencia que establece la primera definición valórica del ser humano. Es el sistema de prestigio y valorización que va a organizar y a estructurar las diferenciaciones básicas de toda sociedad. De la construcción simbólica de la feminidad y la masculinidad arraigada en la cultura depende la percepción de sí mismo como hombre o como mujer y repercute en la autoestima y en la identidad de cada persona, ordenando los comportamientos y delimitando los espacios sociales y el tipo de acciones que ejercerán.

Las personas se construyen a sí mismas asimilando la identidad y la ciudadanía que les confiere su cultura, situándolas en un esquema de prestigio, siendo el género junto a la clase, a la etnia, a la religión, entre otros, un aspecto relevante al momento de definir el prestigio asignado. El desarrollo y estímulo de las emociones media esta construcción, siendo la experiencia de emociones positivas la que impulsa a la acción y la asertividad; así una emoción positiva es proactiva y una emoción negativa inhibe la acción y por tanto al sujeto social.

Los modelos de feminidad y masculinidad llevan implícitos el poder social de mujeres y hombres, donde la ciudadanía 
pasiva, aquella condición ciudadana que se constituye en torno a los derechos ya constituidos y al cumplimiento de los deberes y responsabilidades ciudadanos, a partir de las libertades negativas y de la acción ciudadana individual se asocia a la feminidad y se contrapone a una ciudadanía activa, asociada a la masculinidad, donde hay un ejercicio pleno de la ciudadanía, que implica una orientación al comportamiento proactivo para la vigencia de los derechos alcanzados y en pos de la ampliación de derechos a nuevos temas y actores, privilegiándose la acción colectiva en los espacios públicos (11). Este vínculo con la feminidad genera una autopercepción de las mujeres y de los colectivos femeninos que favorece el ejercicio de una ciudadanía limitada en cuanto al ejercicio del poder y de la autonomía.

Por tanto, el objetivo de esta reflexión es abordar la identidad de la enfermería en el contexto de la ciudadanía y el género.

\section{Ciudadanía cotidiana y Enfermería}

Es importante señalar que se hace una distinción entre la ciudadanía jurídico-formal, como señala Amorós (12) y la ciudadanía cotidiana. Es decir, una ciudadanía sin diferencias de género ni otras condiciones subordinadas. Es el ejercicio pleno de la ciudadanía sustentado en los derechos y deberes de todo ciudadano (12). Debido a que los colectivos ciudadanos están matizados de exclusión informal, expresado en la cotidianidad que contrasta con el poder formal-legal, es en la vida cotidiana donde se articulan el espacio privado y el espacio público, la ciudadanía cotidiana es una condición propia de cada persona y está relacionada con la percepción y la vivencia del derecho a la subjetividad y el deber ciudadano (13); ejercer una ciudadanía cotidiana en libertad implica tener incorporado el ser sujeto de derecho y no solamente ser un enunciado de derecho, es decir, ejercer una ciudadanía sustantiva.
Es aquí, en la ciudadanía cotidiana donde converge esta reflexión con la enfermería en su cotidianidad. El género es consustancial a la enfermería y por tanto afecta la expresión de la ciudadanía que conlleva. Como disciplina, ha ido progresivamente incorporándose en el espacio político de la ciencia. Uno de los factores que ha obstaculizado esta entrada ha sido el género, enraizado con el prestigio otorgado al campo de saber, de tal forma que la vinculación de la enfermería a la feminidad supone una socialización al nopoder, como describe Del Valle (14), lo que dificulta la reivindicación del conocimiento generado por la disciplina de enfermería y su validación como ciencia.

Por otra parte, enfermería se vivencia a través de la interacción social de al menos dos ciudadanos o ciudadanas en una relación de poder que puede ser horizontal en tanto la enfermera o el enfermero tengan interiorizado un quehacer basado en valores humanistas, donde el cuidado se concibe holístico y respetuoso de los derechos a la subjetividad y a la diferencia. Donde el no saber, el no conocer a su interlocutor no sea un obstáculo para reconocer la condición de igualdad, abriendo la posibilidad de descubrir y crear una realidad única y trascendente para la enfermera o enfermero y para la persona que necesita el cuidado.

Pero, en esta interacción que se crea, también puede suceder que la relación de poder sea asimétrica porque la enfermera o el enfermero tienen incorporado el saber experto que les posiciona en ventaja por sobre quien necesita del cuidado de enfermería. Si para la enfermera o el enfermero esta persona no es un sujeto de derecho y la atención se basa en la beneficencia o asistencialismo entonces el trato es vertical e impide el encuentro con la persona.

A la condición anterior se suma la presión de la lógica de empresa que impregna a la salud y a las personas. La enfermería productiva, orientada hacia el cumplimiento de 
metas, es funcional a un sistema económicosocial que despoja a la enfermera o enfermero de su derecho a ejercer su profesión desde la lógica ontológica de su disciplina, para someterlo a una práctica que espera que cada acción implique un producto. Y a quién recibe el cuidado lo cosifica al transformarlo en un caso o cliente, de modo que el o la profesional sólo puede ejercer una ciudadanía pasiva y nominal.

En este punto es importante reflexionar sobre los productos y el impacto de la acción de la profesión. La enfermería tiene el reto de conciliar la exigencia de obtener un resultado positivo a corto plazo. Ser un/a enfermero/a eficiente, en el ejercicio de una enfermería humanista (15), con frecuencia se contrapone, dado que en esta última el impacto de la acción no siempre es identificable ni medible con los instrumentos preestablecidos, $y$ que además ello no es relevante, porque se sabe que el efecto de la enfermería supera la inmediatez y la lógica causal por estar sustentada en una lógica de la complejidad humana. El cuidado recibido alivia el problema de salud específico a la vez que trasciende en otros ámbitos de la persona, el efecto del cuidado es difícil de identificar y anticipar lo que redunda en su invisibilización política, no obstante, el buen ejercicio de la enfermería facilitaría la construcción de una sociedad de mayor igualdad y respeto.

A lo anterior se suma la condición de enfermería de estar sujeta a modelos ajenos a ella que conlleva un costo de identidad y de libertad. A pesar de lo anterior, la enfermería y el cuidado están en un momento de resignificación social, en un proceso único de construcción donde el reto está en afianzar su identidad distanciándose de modelos hegemónicos preconcebidos, de forma que como ciencia y profesión tiene la oportunidad de contribuir al conocimiento situado en el tiempo y en la cultura que retribuya bienestar y mejor calidad de vida.

\section{Enfermería en la tensión del cambio}

La resignificación de la enfermería en el ámbito de la salud está vinculada directa e indirectamente con la sociedad (16). Directamente en tanto el cuidado se ha transformado en un tema social, que ha traspasado las fronteras del espacio privado para posicionarse en el espacio público como un objeto de políticas públicas. La emergencia del cuidado como asunto político está vinculado a las necesidades que tienen los seres humanos para asegurar su supervivencia, los cuidados que se derivan de estas necesidades son entre otros los relacionados al proceso de saludenfermedad de las personas en su trayectoria de vida.

La importancia social de los cuidados tiene al menos dos aspectos claves: Por una parte está el cambio en la economía por la alteración en el sistema de sexo-género, que abre la posibilidad a las mujeres de desarrollar un proyecto de vida propio, diferente al que reconoce el modelo cultural tradicional, transformando el cuidado en un bien escaso debido a la falta de cuidadoras naturales. Esta situación ha visibilizado la importancia de los cuidados para la continuidad de la sociedad, haciéndose indispensable para afrontar la vulnerabilidad del ser humano en los momentos cruciales de su ciclo vital.

Junto a lo anterior hay que resaltar la especialización del cuidado basado en el conocimiento experto, el reconocimiento de la individuación de los sujetos, el paso de la ciudadanía formal-legal a la ciudadanía cotidiana, que aunado al desarrollo y socialización del conocimiento hacen que el cuidado exija un conocimiento específico y situado. Es decir que el saber experto se adapte a la persona contextualizada que necesita el cuidado, en otras palabras, la condición protagónica del sujeto exige un cuidado complejo, de forma que la enfermería, cuyo sentido se centra en el cuidado de las personas en el proceso de salud-enfermedad, se identifica 
como una profesión y disciplina imprescindible para la sociedad, dado que su saber experto se orienta a la persona y a su salud en su contexto social, poseedora de una visión sistémica que exige un saber inteligente, no reducido al saber instrumentalizado.

Esta nueva significación se vincula indirectamente a la sociedad, dado que la consolidación de la disciplina de enfermería exige un reconocimiento social de mayor prestigio que perturba el equilibrio de poder al interior de la ciencia y también en la cotidianidad de la vida. La enfermería es una expresión de las microrrealidades que está enmarcada en una organización y estructura definida sustentadas por una cultura específica, adscribiéndose a las creencias, valores y prejuicios que ordenan simbólicamente a la sociedad.

Aunque esto no es privativo de la enfermería sino que es una condición compartida por todos los saberes y quehaceres humanos, es ella una de las profesiones más estigmatizadas en Occidente por la condición particular que en ella se da: la subordinación de la feminidad (17) en el sistema sexo-género y el correlato de esta subordinación en el saber experto.

El estigma cultural de la enfermería exige a la disciplina un gran esfuerzo adicional respecto a otros saberes para posicionarse legítimamente en la ciencia. Desde la perspectiva del núcleo de la cultura hegemónica, mujer, feminidad y cuidados no son desafíos para indagar y conformar un conocimiento científico. Esta creencia constituye un prejuicio que dificulta el posicionamiento del trabajo que realizan las enfermeras y con mayor crudeza los enfermeros, por ser hombres que optan por una profesión tradicionalmente femenina. El estigma asociado a la feminidad y la naturalización de sus habilidades y saberes en la cultura androcéntrica aumenta cuando un hombre decide ser enfermero.

El prejuicio actúa de forma innata e inconsciente y permite resituar, reordenar la vida cotidiana de forma coherente con las creencias y valores que estructuran la socie- dad. Los prejuicios se viven junto a las emociones que suscitan, en este caso favorecen la infravaloración, la descalificación y la invisibilización.

Hay que agregar que las mujeres y los hombres que optan por la enfermería forman parte también de esta cultura, por tanto en ellas y ellos se confrontan en sus expectativas, creencias y valoraciones que a veces pueden ser antagónicas con la percepción que tienen de la disciplina y de la profesión. Comportándose a veces en consistencia con el orden hegemónico, aceptando el posicionamiento al interior del prestigio social otorgado a la ciencia de enfermería.

Sin embargo, la transgresión de género de la feminidad se observa en el cambio que promueve la enfermería al interior del ámbito de la salud y por tanto de la sociedad. El posicionarse en un nuevo escenario de prestigio implica la confrontación con la cultura hegemónica que en mayor o menor grado las enfermeras y enfermeros también han compartido.

En la vida cotidiana, específicamente en Chile, se observa una brecha entre la práctica de la profesión y la reflexión de la disciplina, las enfermeras en su quehacer cotidiano y el contexto de la salud en el cual trabajan no logran conciliar completamente el cambio producido en la disciplina con el quehacer de la profesión, esto crea una tensión de difícil comprensión y solución. Existe una inercia marcada por la cultura compartida por enfermeras, enfermeros, de los otros profesionales de la salud y de las mismas personas que reciben el cuidado de enfermería, que hace a enfermería enfrentarse con frecuencia a límites difusos.

La fuerte impronta androcéntrica del sistema sexo-género de la cultura chilena hace que la inclusión de hombres en la profesión sea vista como una posibilidad de favorecer el prestigio de la enfermería, siendo una minoría los hombres que optan por ser enfermeros. Es frecuente que las mujeres enfermeras masculinicen en el lenguaje la profesión, 
hablando por ejemplo del saber enfermero o del proceso enfermero, esta buena intención lejos de favorecer la autonomía del conocimiento de enfermería y la validación del esfuerzo histórico y político de las mujeres a través de la reivindicación de la enfermería, beneficia al sistema hegemónico al invisibilizar la fuerza y la cohesión del pensamiento y voluntad de un colectivo subordinado.

Ante la evidente consolidación y justipreciación de la disciplina, el androcentrismo reacciona y se aprecia la implementación de la estrategia de poder conocida como "techo de cristal" (18) para el control de la enfermería, de las enfermeras y enfermeros, limitando sutilmente el empoderamiento de la disciplina al interior de las ciencias y de la sociedad, a través de la obstaculización de la asignación de responsabilidades de salud de alto nivel.

El cambio que la enfermería está protagonizando es cultural, forma parte de la nueva expresión en el orden del sistema sexo-género, siendo una opción de las enfermeras y enfermeros comprometerse con este cambio y conseguir una identidad sólida que articule la práctica con la disciplina, constituyéndose en un sujeto social que transforme la realidad defendiendo la salud de las personas como un derecho inalienable.

\section{REFERENCIAS}

1. Moore H. Antropología y Feminismo. Madrid: Cátedra; 1996.

2. Pavez A. La construcción del sujeto social: una articulación de la identidad, la ciudadanía y el género. [Tesis doctoral]. España: Universidad del País Vasco/Euskal Herriko Unibertsitatea; 2010.

3. Zúñiga $Y$, Paravic T. El género en el desarrollo de la enfermería. Rev Cubana Enfermer [revista en la Internet]. 2009 Jun [citado 25 oct 2013]; 25(1-2): Disponible en: http://scielo.sld.cu/
4. Chamizo C. La perspectiva de género en Enfermería: comentarios y reflexiones. Index Enferm. 2004; 13(46): 40-44.

5. Santo T, Oguisso T, Fonseca R. La profesionalización de la enfermería brasileña en los medios de comunicación escritos de final del siglo XIX: un análisis de género. Rev Lat Am Enfermagem. 2011; 19 (5): 1265-1271.

6. Mead, G. H. Espíritu, persona y sociedad, Buenos Aires: Paidós; 1968.

7. Pérez Cantó P. (ed.) También somos ciudadanas. Madrid: Universidad Autónoma de Madrid, Instituto Universitario de Estudios de la Mujer; 2000.

8. Del Valle T. et al. Modelos emergentes en los sistemas y relaciones de género. Madrid: Narcea; 2002.

9. González S, Cavieres H, Díaz C, Valdebenito M. Revisión del constructo de Identidad en la Psicología Cultural. Revista de Psicología. 2005; 14(2): 9-25.

10. Thurén Britt M. El poder generizado. Madrid: Instituto de investigaciones feministas; 1993.

11. González S. La noción de ciudadanía en jóvenes estudiantes secundarios y universitarios: un análisis de estudios comparados de la nueva ciudadanía. En Zambrano, Rozas, Magaña, Asún y Pérez-Luco, editores: Psicología comunitaria en Chile: evolución, perspectivas y proyecciones. Santiago: RIL Editores; 2007.

12. Amorós C. El espacio de los iguales, espacio de las idénticas. Notas sobre poder y principio de individuación. Arbor. 1987: 113-127.

13. Pavez A. Género y emociones en el ejercicio de la ciudadanía cotidiana. En Gregorio $\mathrm{C}$, Castañeda $\mathrm{P}$, editores: Mujeres y hombres en el mundo global. México: Siglo XXI; 2012.

14. Del Valle T. Mujer y nuevas socializaciones: su relación con el poder y el cambio. KOBIE. 1992/1993; 6: 2-15.

15. Sanhueza O. Contribución de la investigación cualitativa a enfermería. Cienc. 
enferm. 2009; XV(3): 15-20.

16. Porto F, Amorim W. Historia da enfermagem: identidade, profissionalização e símbolos. São Caetano do Sul: Yendis; 2010.
17. Lillo Crespo M. Antropología, género y enfermería contemporánea. Rev Enferm. 2002; 25(12): 856-862.

18. Valcárcel A. La política de las mujeres. Madrid: Cátedra; 1997. 